\title{
Christof Stock, Barbara Schermaier-Stöckl, Verena Klomann, Anika Vitr (2020). Soziale Arbeit und Recht. Fallsammlung und Arbeitshilfen
}

\section{2., aktualisierte und erweiterte Auflage, Baden-Baden: Nomos Verlagsgesellschaft. ISBN 978-3-8487-6926-1, 248 S., $34 €$}

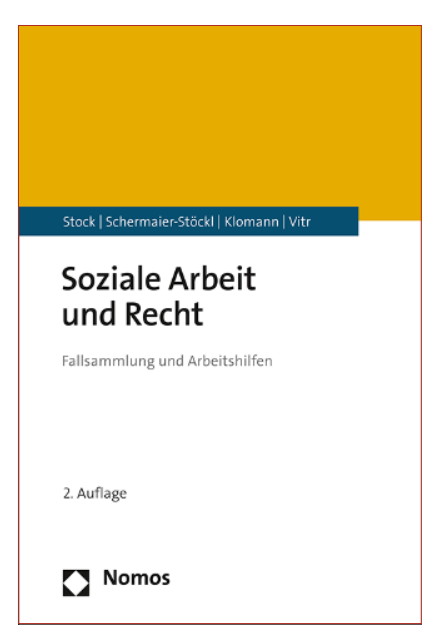

nenteam setzt sich aus zwei Professor innen für Recht in der Sozialen Arbeit, einer Professorin für Theorien und Konzepte Sozialer Arbeit sowie einer Rechtsanwältin und Lehrbeauftragten an der Katholischen Hochschule Nordrhein-Westfalen zusammen.

Das Lehrbuch ist in zehn Kapitel untergliedert und nimmt die wesentlichen Themenfelder der Sozialen Arbeit in den Blick. Die einzelnen Kapitel sind vergleichbar strukturiert, indem sie anhand von Praxisfällen die vielschichtigen Handlungsanforderungen an Sozialarbeiter_innen reflektieren und die einzelnen Fälle hinsichtlich ihrer rechtlichen Aspekte lösen.

In einem einführenden Kapitel erhalten die Leser_innen zunächst allgemeine Hinweise zum Umgang mit juristischen Texten, bevor sie anschließend in einem professionstheoretischen Diskurs für das Verhältnis zwischen dem Recht als Bezugswissenschaft einer disziplinären und professionellen Sozialen Arbeit sensibi- lisiert werden. Das zweite Kapitel befasst sich mit der Rechtsordnung in ihrer abstrakten Struktur und diskutiert unter anderem wesentliche Fragestellungen des Zivil- und öffentlichen Rechts, die Grundgedanken der Rechtsgebiete Zivil-, Verwaltungs- und Strafrecht oder das sozialhilferechtliche Dreiecksverhältnis zwischen Leistungserbringer, Leistungsträger und Leistungsempfänger. Hierbei werden konstitutive Grundsätze des allgemeinen Rechtssystems vermittelt, welche sich in den nachfolgenden Themenfeldern der Sozialen Arbeit durchgehend wiederfinden. Die nachfolgenden Themenfelder der Sozialen Arbeit widmen sich vorwiegend den komplexen Lebenswelten und Problemlagen ihrer Adressat_innen und betrachten im weiteren Verlauf die rechtlichen Rahmenbedingungen sozialer Hilfen sowie sozialarbeiterischer Beratungs- und Unterstützungsangebote. Zunächst wird die Soziale Arbeit im Kontext finanzieller Problemlagen in den Blick genommen und wesentliche Bausteine der Existenzsicherung vorgestellt. Dabei wird das Verhältnis des Zivil- und öffentlichen Rechts sowie die sozialstaatlichen Leistungen der Grundsicherung und Sozialhilfe näher betrachtet. Nachfolgend wird die Soziale Arbeit mit Paaren, Familien, Kindern und Jugendlichen fokussiert. Neben der umfassenden Darstellung familienrechtlicher Grundlagen wie Abstammung oder das Sorge- und Umgangsrecht, werden die sozialstaatlichen Rechtsgrundlagen der Kinder, Jugend- und Familienhilfe aufgegriffen. Durch die verfassungsrechtliche Reflexion der Beziehung zwischen dem Elternvorrang in der Pflege und Erziehung von Kindern sowie dem Schutzauftrag des Staates für Kinder und Jugendliche wird gleich zu Beginn des Kapitels das klassische Spannungsverhältnis von „Hilfe und Kon- 
trolle“ im Kontext ihrer differenzierten Handlungsanforderungen an die Soziale Arbeit dargestellt. Hierbei werden im weiteren Verlauf des Kapitels die rechtlichen Anspruchsgrundlagen und Handlungsverpflichtungen der sozialstaatlichen Unterstützungs- und Kontrollfunktion Sozialer Arbeit näher betrachtet. Im Kapitel zur Berufsausübung in der Sozialen Arbeit beantwortet das Lehrbuch beispielsweise Fragen zu unterschiedlichen Beschäftigungsverhältnissen, der Schweigepflicht oder persönlichen Haftung von Sozialarbeiter_innen. Weitere Themenfelder sind die Soziale Arbeit und Bildung, die sich mit den Ansprüchen und der Förderung von früher Bildung bis zur Erwachsenenbildung beschäftigt, die Soziale Arbeit und Behinderung sowie die Soziale Arbeit im Gesundheitswesen. Auch wird die Soziale Arbeit im Kontext von Migration und Flucht behandelt und neben den Grundlagen zum Staatsangehörigkeitsrecht, wesentliche Kenntnisse zum Ausländerrecht im engeren Sinne sowie dem Asylverfahren vermittelt. Abschließend widmet sich das Lehrbuch der Sozialen Arbeit im Kontext von Gewalt und Straffälligkeit. Dieses Kapitel gliedert sich in die Arbeit mit jugendlichen und erwachsenen Straffälligen sowie Betroffenen von Gewalt und Straftaten. Hierbei werden unter anderem die sozialarbeiterischen Handlungsanforderungen der Jugendhilfe im Strafverfahren hinsichtlich ihrer rechtlichen Rahmenbedingungen betrachtet.

Im Gegensatz zu anderen Professionen lässt sich der Gegenstand der professionellen Interaktionen meist nicht ausschließlich auf ein Themenfeld reduzieren. Grundsätzlich kann jede Lebenssituation von Menschen zum Gegenstand Sozialer Arbeit werden, sofern sie durch die Adressat_innen oder professionellen Fachkräfte (z. B. im Kontext des staatlichen Wächteramts) als problematisch und veränderungsbedürftig beschrieben wird. Sozialarbeiter_innen müssen folglich oftmals auf rechtliches Wissen unterschiedlicher Themenfelder zurückgreifen und dieses im weiteren Verlauf professionell ausgestalten. Dieser sogenannten „Allzuständigkeit" Sozialer Arbeit wird das vorliegende Lehrbuch gerecht, indem es die interdisziplinären Perspektiven der Autor_innen vereint und neben der Vermittlung grundlegender Kenntnisse des Rechtssystems die oftmals unbestimmten Rechtsbegriffe sozialarbeiterisch definiert. Als besonders hilfreich erweist sich dabei die didaktische Darstellung der unterschiedlichen Praxisfälle, welche den Leser_innen einen lebendigen und schnellen Einstieg in die unterschiedlichen Themenfelder ermöglicht. Das Lehrbuch eignet sich somit hervorragend zur systematischen Einarbeitung in das Verhältnis von Sozialer Arbeit und Recht sowie als Nachschlagewerk. Dabei erfüllt es durchgehend den Anspruch, Sozialarbei- ter_innen - und keine Jurist_innen - auszubilden und leistet einen wertvollen Beitrag zur Etablierung einer professionellen Sozialen Arbeit.

Eingegangen. 3. Mai 2021

Angenommen. 3. Mai 2021

Funding. Open Access funding enabled and organized by Projekt DEAL.

Open Access. Dieser Artikel wird unter der Creative Commons Namensnennung 4.0 International Lizenz veröffentlicht, welche die Nutzung, Vervielfältigung, Bearbeitung, Verbreitung und Wiedergabe in jeglichem Medium und Format erlaubt, sofern Sie den/die ursprünglichen Autor(en) und die Quelle ordnungsgemäß nennen, einen Link zur Creative Commons Lizenz beifügen und angeben, ob Änderungen vorgenommen wurden.

Die in diesem Artikel enthaltenen Bilder und sonstiges Drittmaterial unterliegen ebenfalls der genannten Creative Commons Lizenz, sofern sich aus der Abbildungslegende nichts anderes ergibt. Sofern das betreffende Material nicht unter der genannten Creative Commons Lizenz steht und die betreffende Handlung nicht nach gesetzlichen Vorschriften erlaubt ist, ist für die oben aufgeführten Weiterverwendungen des Materials die Einwilligung des jeweiligen Rechteinhabers einzuholen.

Weitere Details zur Lizenz entnehmen Sie bitte der Lizenzinformation auf http://creativecommons.org/licenses/by/4.0/deed.de. 